San Jose State University

SJSU ScholarWorks

\title{
The Effect of Basal Bolus Insulin Administration vs. Sliding Scale Insulin on Glycemic Control in Patients with Type 2 Diabetes Mellitus at a Community Hospital
}

\author{
Meriam Signo \\ California State University, Northern California Consortium Doctor of Nursing Practice
}

Follow this and additional works at: https://scholarworks.sjsu.edu/etd_doctoral

Part of the Other Nursing Commons, and the Perioperative, Operating Room and Surgical Nursing Commons

\section{Recommended Citation}

Signo, Meriam, "The Effect of Basal Bolus Insulin Administration vs. Sliding Scale Insulin on Glycemic Control in Patients with Type 2 Diabetes Mellitus at a Community Hospital" (2019). Doctoral Projects. 94. DOI: https://doi.org/10.31979/etd.ecat-qcw3

https://scholarworks.sjsu.edu/etd_doctoral/94

This Doctoral Project is brought to you for free and open access by the Master's Theses and Graduate Research at SJSU ScholarWorks. It has been accepted for inclusion in Doctoral Projects by an authorized administrator of SJSU ScholarWorks. For more information, please contact scholarworks@sjsu.edu. 


\section{ABSTRACT \\ THE EFFECT OF BASAL BOLUS INSULIN ADMINISTRATION VS. SLIDING SCALE INSULIN ON GLYCEMIC CONTROL IN PATIENTS WITH TYPE 2 DIABETES MELLITUS AT A COMMUNITY HOSPITAL}

Evidence of effective insulin delivery by nurses can help prevent fluctuating plasma glucose levels of Type 2 diabetes mellitus (T2DM) in the hospital setting. Information leading to better diabetes care using either basal bolus insulin administration (BBI) or sliding scale insulin (SSI) is essential for safe blood sugar values. The purpose of this project was to measure patient glycemic control after the implementation of BBI vs. SSI during a patients' stay in the hospital. This study was a quantitative, retrospective exploratory chart review of T2DM in medical-surgical units at a Northern California community hospital. The data were collected from routine standardized point of care testing documentation on an electronic record. The study timeframe of chart reviews occurred from May 2017 to May 2018. Results suggest that SSI provided better glycemic control in hospitalized patients with T2DM. This study showed when using SSI, the average blood glucose was lower and that the estimated mean HgbA1c was lower in comparison to $\mathrm{BBI}$.

Meriam Signo

May 2019 

THE EFFECT OF BASAL BOLUS INSULIN ADMINISTRATION VS. SLIDING SCALE INSULIN ON GLYCEMIC CONTROL IN PATIENTS WITH TYPE 2 DIABETES MELLITUS AT A COMMUNITY HOSPITAL

by

Meriam Signo

\author{
A project \\ submitted in partial \\ fulfillment of the requirements for the degree of \\ Doctor of Nursing Practice \\ California State University, Northern Consortium \\ Doctor of Nursing Practice
}

May 2019 


\section{APPROVED \\ For the California State University, Northern Consortium Doctor of Nursing Practice:}

We, the undersigned, certify that the project of the following student meets the required standards of scholarship, format, and style of the university and the student's graduate degree program for the awarding of the Doctor of Nursing Practice degree.

Meriam Signo

Project Author

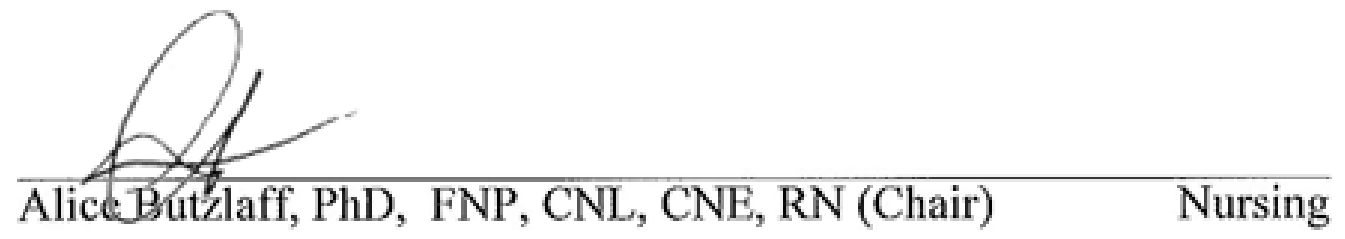

Cotian (a) isor

Catherine Carson, MPA, BSN, RN, CPHQ El Camino Hospital

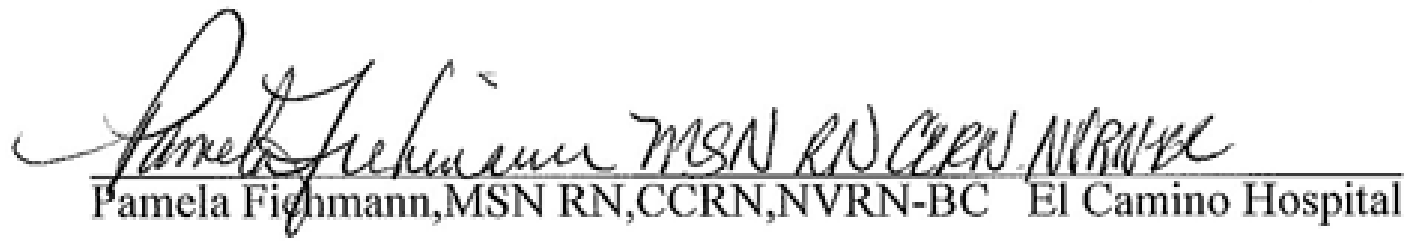


AUTHORIZATION FOR REPRODUCTION

OF DOCTORAL PROJECT

I grant permission for the reproduction of this project in part or in its entirety without further authorization from me, on the condition that the person or agency requesting reproduction absorbs the cost and provides proper acknowledgment of authorship.

Permission to reproduce this project in part or in its entirety must be obtained from me.

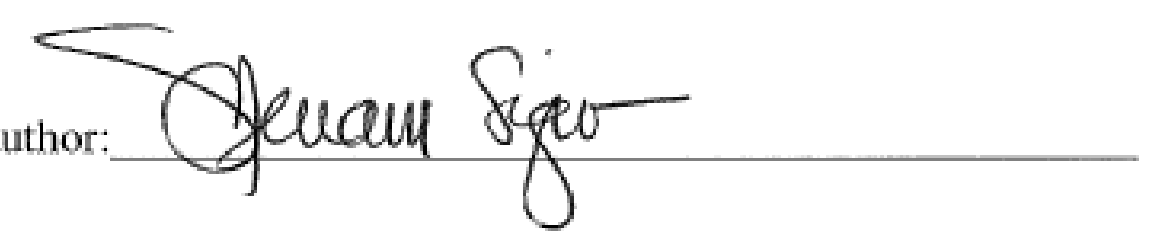




\section{ACKNOWLEDGMENTS}

Going back to pursue a Doctor Nursing Practice degree has been a journey. The journey has been both meaningful and rewarding to me. First, I want to thank my family, Chito and Alli. They are definitely above and beyond amazing, such blessings!

Second, I want to thank Dr. Alice Butzlaff, my project chair who provided guidance, support, and her clinical expertise in diabetes. I want to thank the following advisors: Dr. Nisha Nair, Dr. Hilda Pinzon-Perez, Dr. Kammi Sayaseng, and Dr. Ricardo Ballin. Their facilitative support made this journey successful. Special thanks to Dr. Sulekha Anand for your advised on the statistical analysis. Special thanks to the California State University Fresno Graduate Statistics as well.

Third, special thanks to my colleagues at El Camino Hospital. I want to thank Catherine Carson, my mentor and project member - without you, I will not be able to get where I am today. You are the anchor. Thank you, Pamela Fiehmann, for your guidance, support, and you kept me focused and your positivity in me. And, my colleagues at ECH Los Gatos Campus.

Fourth, I want to thank the following scholarship grantors: ECH Auxiliary Foundation, Association of California Nurse Leaders -Alpha Consulting Group, CSU Fresno Scholarships (Sade Smith and Anna Biehl).

There are many who supported me in this journey. I want to say, from my heart to your heart thank you. 


\section{TABLE OF CONTENTS}

Page

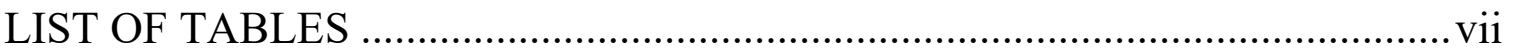

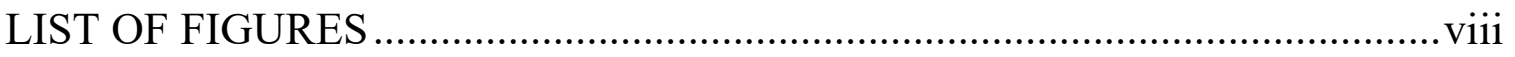

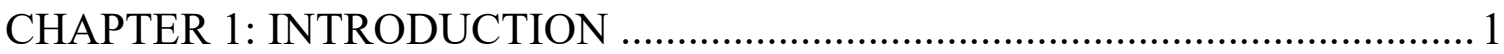

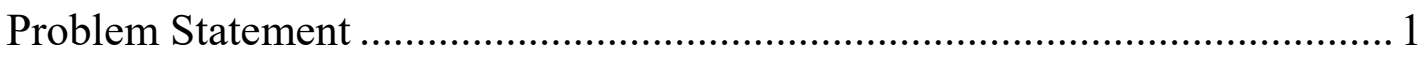

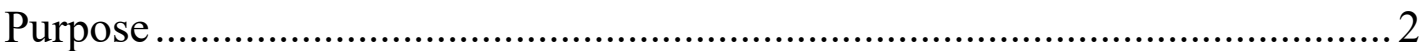

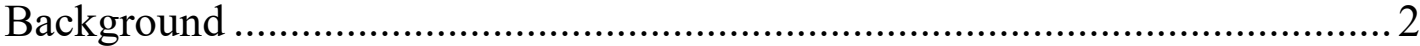

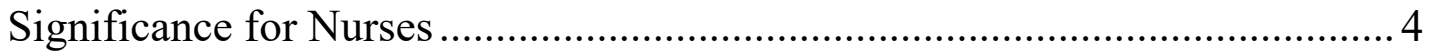

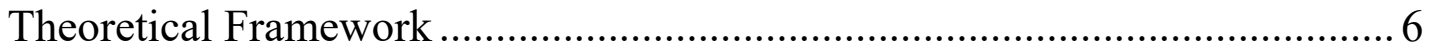

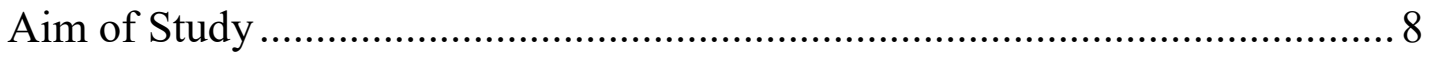

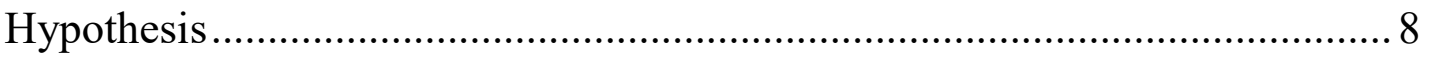

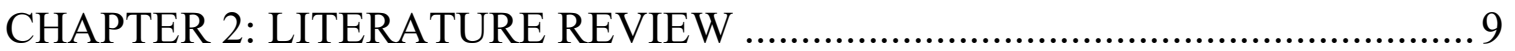

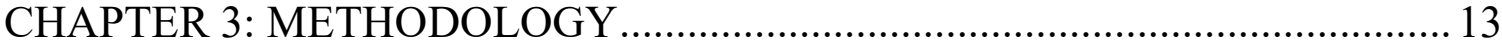

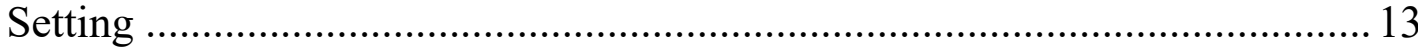

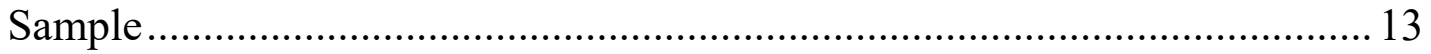

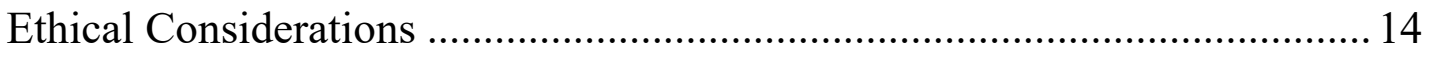

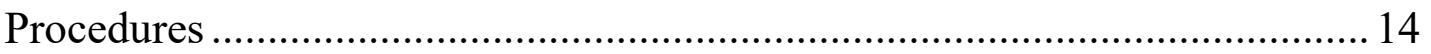

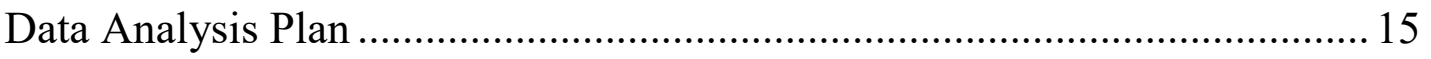

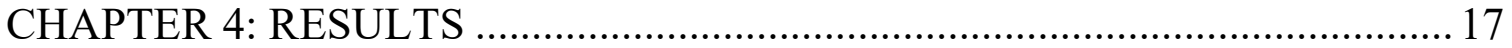

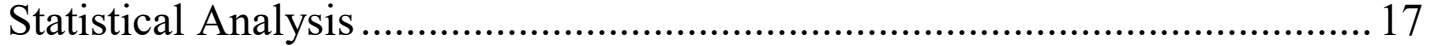

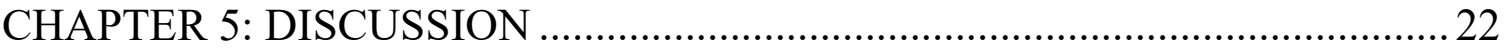

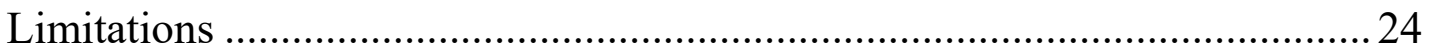

Recommendations for Future Research ………............................................2 24

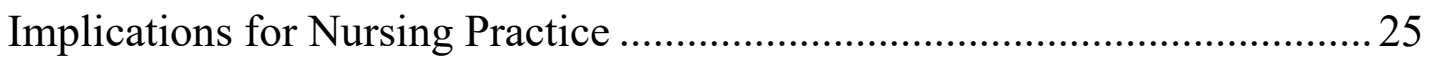


Page

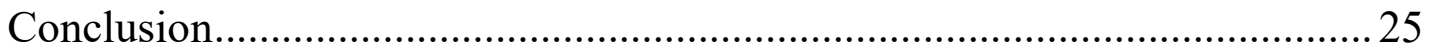

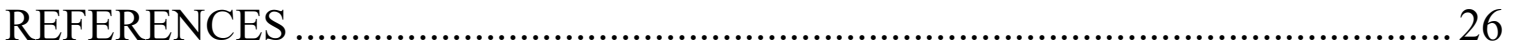

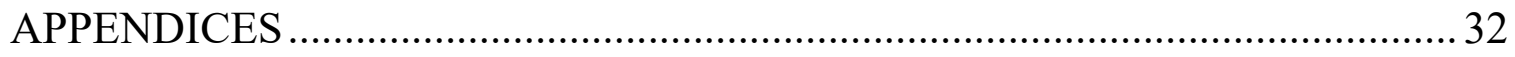

APPENDIX A: EL CAMINO HOSPITAL IRB APPROVAL ............................ 33

APPENDIX B: CALIFORNIA STATE UNIVERSITY, FRESNO IRB

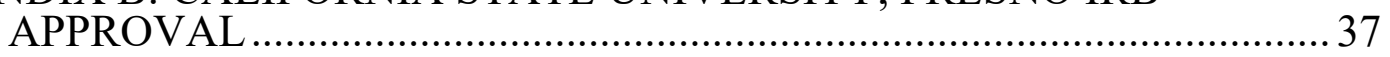




\section{LIST OF TABLES}

Page

Table 1 Descriptive Statistics for Age and Length of Stay for the Entire

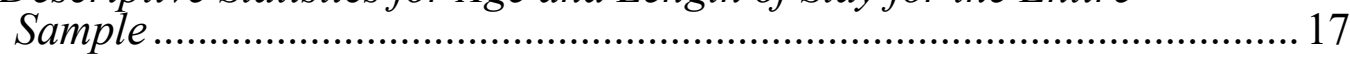

Table 2 Descriptive Statistics for Age and Length of Stay by Group..................... 18

Table 3 Frequencies and Percentages of Gender for the Entire Sample ............... 18

Table 4 Frequencies and Percentages of Gender by Group ................................. 19

Table 5 Independent Samples $t$ Tests for Averages Blood Glucose and

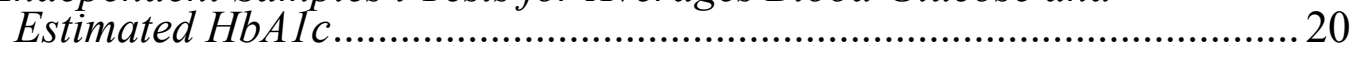




\section{LIST OF FIGURES}

Page

Figure 1. Average blood glucose level mg/dL comparing basal bolus insulin

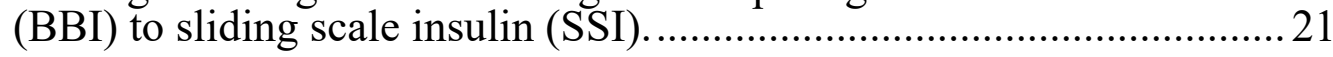

Figure 2. Estimated $\mathrm{HbA1c}$ comparing basal bolus insulin (BBI) to sliding scale insulin (SSI) 


\section{CHAPTER 1: INTRODUCTION}

Diabetes mellitus is a life-threatening disease with chronic long-term complications affecting over 30 million people (Centers for Disease Control and Prevention [CDC], 2017). Ninety-five percent of individuals with diabetes are diagnosed with type 2 diabetes mellitus (T2DM) and 5\% have type 1 diabetes. In 2015, diabetes became the seventh leading cause of death and the cost of the disease in the United States was estimated at \$245 billion (CDC, 2017). Controlling insulin levels for diabetes is more challenging when patients are in the hospital; insulin needs increase during acute illness or surgery because of the physiological stress in the human body (Johnson \& Van Horn, 2011). Ensuring better glycemic control in T2DM is paramount to long-term survival.

\section{Problem Statement}

After the implementation of Basal Bolus Insulin Administration (BBI) guidelines at a Northern California community hospital, concerns were raised if BBI would have better glycemic control in patients with T2DM than Sliding Scale Insulin (SSI). In 2016, the American Diabetes Association (ADA) strongly discouraged only the use of sliding scale insulin in patients with T2DM. Instead, a basal plus bolus correction insulin regimen was the preferred treatment (ADA, 2016). Although several researchers examined the management of T2DM patients in the hospital setting, data on glycemic control has been difficult to record and track for BBI vs. SSI at a Northern California community hospital. There has been minimal research comparing glycemic control between patients managed with $\mathrm{BBI}$ and SSI within the same hospital setting. The aim of this research was to 
retrospectively review blood sugar readings between T2DM patients managed with either BBI vs. SSI.

\section{Purpose}

The purpose of the study was to assess glycemic control following the implementation of BBI as compared to SSI for hospitalized patients with T2DM at a community hospital.

\section{Background}

Diabetes is a metabolic disease characterized by abnormal insulin secretion. Type 2 diabetes mellitus (T2DM) is characterized by insufficient insulin production. According to the American Diabetes Association (2014), T2DM patients have an impaired and defective insulin secretion and response.

Basal bolus insulin administration (BBI) has been defined as when a patient receives a long-acting insulin and a short-acting insulin to minimize abnormal blood glucose levels (Umpierrez et al., 2007). For example, a patient would receive a daily long-acting insulin and before meals rapid-acting insulin analogs (Umpierrez et al., 2007). One disadvantage would be daily multiple injections. Evidence suggests that using BBI improves glucose control, decreases hospital complications, and reduces the length of stay. Umpierrez et al. (2011) found that the application of basal-bolus insulin to T2DM patients after surgery and to noncritically ill patients showed better management of diabetes than sliding scale insulin (SSI), a method discovered in the late 1970s for short-term use in the hospital that was "never associated with improved clinical outcomes" (Umpierrez et al., 2007, p. 2185). Basal-bolus insulin showed improvement in managing diabetes, specifically by preventing hyperglycemia while patients were in the 
hospital (Umpierrez et al., 2011) and decreasing complications associated with diabetes (Harris, Narayan, \& Ali, 2015).

On the other hand, sliding scale insulin (SSI) is the oldest form in the treatment of diabetes. In the sliding scale insulin method, the dose is based upon the blood sugar level just before the next meal. The amount of insulin is listed in a chart. It is based on the progression of increasing the rapid-acting or regular insulin with the addition of scheduled insulin injections to sustain blood glucose levels if required. The major disadvantages of using a sliding scale are that meals should contain the same amount of carbs. In the hospital setting, meals may be regulated by carbohydrate counts, but withholding food, changes in activity and stress will also affect blood glucose levels. Despite disadvantages, this treatment is commonly used in the hospital setting (Alfehaid, Alotaibi, Alanazi, Bustami, \& El Malik, 2018). The principal difference between the two strategies is that slidingscale insulin does not deliver adequate glycemic control to patients and addresses hyperglycemia after it has occurred, whereas a basal-bolus regimen is directed at preventing hyperglycemia (Badlani et al., 2014).

Glycemic control was defined by the American Diabetes Association's (ADA, 2018) official recommendations for people with diabetes-namely, (1) fasting glucose is $80-130 \mathrm{mg} / \mathrm{dL}(4.4-7.2 \mathrm{mmol} / \mathrm{L})$ and (2) 2 hours after meals it is less than $180 \mathrm{mg} / \mathrm{dL}(10.0 \mathrm{mmol} / \mathrm{L})$. Hypoglycemia was defined as follows:

Level 1: A glucose alert value of $3.9 \mathrm{mmol} / 1$ (70 mg/dl) or less.

Level 2: A glucose level of $<3.0 \mathrm{mmol} / 1$ ( $<54 \mathrm{mg} / \mathrm{dl}$ ) is sufficiently low to indicate serious, clinically important hypoglycemia.

Level 3: Severe hypoglycemia, as defined by the American Diabetes Association (2018) denotes severe cognitive impairment requiring external assistance for recovery. 
Glycemic control is important to the patient, nurses, and the hospital for various reasons. For the patient, good glycemic control with proper insulin treatment minimizes microvascular complications, decrease cardiovascular complications, and all-cause mortality. For the purpose of this study, hypoglycemia was measured based on blood glucose levels less than $70 \mathrm{mg} / \mathrm{dL}$ (American Diabetes Association, 2014). Lower blood glucose levels are often not reported as much as higher blood glucose levels. It is important to have glycemic control for T2DM patients to prevent microvascular and cardiovascular complications and to decrease all-cause mortality (Elliott, Fidler, Ditchfield, \& Stissing, 2016). The most common feared adverse effect of diabetes insulin therapy management is hypoglycemia. It is through proper management of treatment modality to achieve good glycemic control ((Elliott et al., 2016).

Point-of-care testing (POCT) is defined as diagnostic type of test done at the patient's bedside and considered as a way to provide quick information about the patient's blood glucose levels (Klonoff, 2014). The recommended general glycemic target is a glycated hemoglobin (HbA1c) level of $<7 \mathrm{mg} / \mathrm{dL}$ (American Diabetes Association, 2016). The estimated glycated hemoglobin (eAG) is a new recommended term from the American Diabetes Association, this allowed reporting of $\mathrm{HbA} 1 \mathrm{c}$ results for diabetes patients, utilized same units $(\mathrm{mg} / \mathrm{dL}$ or $\mathrm{mmol} / \mathrm{L}$ in day-to-day blood glucose measurements (American Diabetes Association, n.d.).

\section{Significance for Nurses}

Nurses specializing in diabetes are key members of the inpatient care team for the translation of research related to evidence-based diabetes practice. In May 2016, a Northern California community hospital conducted a study to understand 
nurses' baseline diabetes patient care knowledge prior to making a treatment change to BBI. Results showed that continued emphasis to improve and sustain nursing knowledge on basal-bolus insulin therapy was necessary.

Recommendations included a larger enterprise-wide education program to introduce basal-bolus insulin therapy as an ongoing educational opportunity in order to sustain nursing knowledge gains and improve confidence levels.

In addition to providing this evidence, nurse practitioners specializing in diabetes should be included on committees that write institutional guidelines and policy on when and how to initiate and change/adjust insulin therapy. The clinical management of T2DM patients in the medical-surgical units is important to the hospital. The importance of glycemic control during the patients' hospital stay improves patients' care outcomes, decreases mortality and morbidity rates, and decreases readmissions and length of stay. Patients recover from their medical illness and surgical patients experience better healing in surgical patients.

The Centers for Medicare and Medicaid Services (CMS) has been working on improving quality care. CMS will focus on healthcare of Medicare beneficiaries, and the quality measures that were helpful for quantifying processes, outcomes, and patient perceptions. Recently, CMS developed a new way of identifying and prioritizing measures called "Meaningful Measures" (National Quality Forum [NQF], n.d.). The focus was to develop new medication measures with the chance of being validated by the National Quality Forum (NQF), an organization aimed at evaluating and supporting standardized performance measurement. Two of the Hospital Inpatient Quality Reporting (IQR) with clinical quality measures involve inpatients: (a) the NQF 2362, which is glycemic controlhyperglycemia and (b) NQF 2363, which is glycemic control-severe hypoglycemia (NQF, n.d.). 
Another goal of conducting this project was to contribute to quality improvement of the NQF performance standards within a Northern California community hospital organization. By applying measure NQF 2363, the metrics of the BBI and SSI were measured. The goal would be to raise awareness about providing high-quality care for T2DM patients reporting glycemic control while hospitalized.

\section{Theoretical Framework}

The implementation of BBI in a community hospital setting involves multiple layers of systems. In fact, the use of BBI was a patient care outcome project related to quality improvement initiatives. The investigator used two theories for studying this phenomenon: (a) general systems theory by Ludwig von Bertalanffy (1972) and (b) complexity theory, which was based on general systems theory (Skyttner, 2006).

Complexity theory sees the organization as the system and the subsystems and their interactions with the processes, outcomes, and foreseeable and unforeseeable consequences that may occur over time (Chandler, Rycroft-Malone, Hawkes, \& Noyes, 2015). According to Johnson (2011), complexity theory explains how the behavior of the whole, rather than its parts, result in complex behaviors. He defined the theory as "the study of phenomena which emerge from a collection of interacting objects" (section 1.1). Based on this concept, communication between systems and subsystems is crucial when dealing with any changes in clinical practices that involve patients. The advantage of complexity theory is that it brings people together, generate debate on ideas, and develop a framework designed to explain the organization's changes. This approach involves engaging disciplines when a new "attractor" is introduced and how the behaviors 
of those affected are acknowledged. Tuffin (2016) mentioned how vital it is to collaborate rather than imposing top-down leadership. It is essential to provide sufficient feedback and allow agreeable solutions within the organization.

It is often a challenge to convince clinicians to use a new model of care that is evidence-based practice. The complexity theory assumes that any actions performed are best accomplished by working on the barriers as a cohesive group. According to Litaker et al. (2006), the group needs to consider the general application of the clinical practice as well as its effectiveness in guiding care management in addition to recognizing different perspectives. Interdisciplinary relationship sessions are recommended, such as performing the assessment of the application, identifying the domains, and building methods together.

According to Cooper and Geyer (2009), although diabetes has been known as a complex disease, few clinicians focus on using a theoretical framework to guide practice. Complexity theory has been criticized and considered as an "allinclusive nature of complexity seeing it as an intangible theory that fails to offer new [ideas] to health care" (Cooper, 2009, p. 761). Some clinicians acknowledge that the use of the old methodology in managing complex diseases failed because a theoretical framework was not used and the interaction within the system was not addressed. Based on two separate studies done by The Action to Control Cardiovascular Risk in Diabetes Study Group (2008) and van der Steeg et al. (2008), low glucose levels in high-risk T2DM patients were not recognized, placing the diabetes population at greater risk of developing coronary artery disease. The emergent results revealed the consequences of failing to recognize the harmful effects of poor glycemic control. If complexity theory had been used, this could have been prevented. Complexity theory allows for unpredictability and 
uncertainty and it permits learning and discovery within the system, thereby enabling complexity thinking to occur (Cooper \& Geyer, 2009).

What is important to know is that the critical system's "attractors" are important stakeholders who should be engaged at the start of the assessment and throughout the evaluation phase. Complexity theory allows for unpredictability. Using complexity theory allows the clinical practice and the theory to come together, resulting in more significant outcomes, such as the prevention of hyperglycemic and hypoglycemic events during patients' hospital stay. BBI requires constant evaluation to ensure that the process would be practical and realistic in the system.

\begin{abstract}
Aim of Study
The aim of the project was to assess glycemic control following BBI in comparison to SSI in the management of T2DM in hospitalized patients.
\end{abstract}

\title{
Hypothesis
}

The hypothesis was that there is a significant relationship between BBI vs. SSI for glycemic control in hospitalized patients with T2DM. 


\section{CHAPTER 2: LITERATURE REVIEW}

A literature review was undertaken to examine the efficacy of BBI and SSI in the management of hospitalized T2DM patients. The investigator searched databases for relevant literature on: T2DM, glycemic control, hypoglycemia, hyperglycemia, insulin therapy, basal-bolus and sliding scale. First, research on T2DM relevant to the effects of insulin therapy were examined. Second, studies that involved insulin therapy provided to T2DM patients in a hospital setting were reviewed. Third, the investigator focused on the use of BBI compared to SSI. Four studies were selected (Fahim et al., 2015; Roberts \& Godbole, 2015; Rymaszewski \& Breakwell, 2013; Umpierrez et al., 2013).

Fahim et al. (2015) conducted a prospective multiphase study to assess newly conducted $\mathrm{BBI}$ dosing guidelines in the medical unit before implementing BBI hospital-wide. The dosing guidelines were designed to check the blood glucose (BG) levels of patients with T2DM then compare BG before and after receiving BBI. The four phases of the study were as follows: (a) review of BBI guidelines nationwide; (2) conduct multidisciplinary clinical staff education on the dosing guidelines; (3) enroll 43 patients in the pilot study; and (4) conduct data analysis using chi-square for categorical variables, paired t-test for continuous variables, and descriptive statistics for determining demographics. Results indicated that the total BG was $377 \mathrm{mg} / \mathrm{dl}$ values before the use of dosing guidelines and $723 \mathrm{mg} / \mathrm{dl}$ values after.

Fahim et al. (2015) noted a statistically significant difference in the BG parameters between pre and post BBI at the end points; the pre BBI was $\mathrm{p}<0.5$ and the post BBI was $\mathrm{p}<0.05$. There was no occurrence of diabetic ketoacidosis or hyperosmolar hyperglycemic states. The authors revised the insulin dosing 
guideline parameters and the units per kilogram and nurses provided insulin administration based on the dosing guidelines. The strength of the study was the multiphase approach, which identified the $\mathrm{BG}$ guidelines needed to be changed based on the results of the pilot phase. Conclusions supported having all physicians adjusting insulin doses based on the BBI guidelines.

Roberts and Godbole (2015) also conducted a retrospective and prospective chart review to identify the accuracy of BBI and SSI dosing administration in a tertiary teaching hospital. The retrospective chart review was done for the SSI and a prospective chart review for the BBI. The sample population was 46 diabetic inpatients with less than 5 days of receiving BBI. Investigators only studied and analyzed insulin given at 0700,1200 , and 1700 . The 2100 hours insulin administration blood glucose levels were not analyzed because it will be gone by the next blood glucose check at 0700. The intervention was as follows: (1) the BBI chart was used to document blood glucose levels and had detailed information of the ordered insulin dosages; and (2) the SSI protocol was in the patient medication administration record, which required the nurses to document the blood glucose in different forms.

Roberts and Godbole (2015) used a chi-square to measure the correlation of the doses that were not given and $t$ tests to correlate the results. Results showed more missed SSI doses than BBI, and 41 missed doses (61.3\%) of SSI as compared to the 12 medication doses of BBI $(\mathrm{p}<.001)$. The SSI missed doses mainly pertained to the group at 2100 , while the BBI was $0700,1200,1700$, and 2100 -hour times. They found that low doses of SSI (8.7\%) and BBI (8.8\%) was almost the same at $\mathrm{p}=.96)$. SSI patients $(2.3 \%)$ received higher medication doses as compared to BBI patients $(\mathrm{p}=.004)$. The limitation of the study was the retrospective chart review; it did not provide a full data capture on missed doses, 
uncertain if missed, documentation or no medication available. The strength of this study was the use of BBI regimen format in the workflow making it easier to identify missed doses in one sheet as compared to SSI where separate sheets were required to document blood glucose levels and the insulin medications.

A classic retrospective chart review conducted by Rymaszewski and Breakwell (2013), the differences between the BBI and SSI were investigated using a convenience sample of $128 \mathrm{~T} 2 \mathrm{DM}$ in-patients who were between 18-80 years old in the medical units at a teaching hospital. They reviewed BBI and SSI regimens of patients who did not eat, abnormal glucose levels in the blood, length of stay (LOS) and events of hypoglycemia. Patients were excluded if they received steroids, were on oral diabetic medications or intravenous insulin, had surgery, or were in the hospital for less than 3 days. There were three goals in this study. The first goal was to determine the significant difference in blood glucose levels between BBI and SSI on fasting, mean and discharge. The difference was supported by the fasting blood glucose values, which showed SSI were higher than BBI (SSI 201.4; BBI 135.5; $\mathrm{p}<.001$ ), the mean blood glucose values were higher in SSI than BBI (SSI 225; BBI 149; p,.001), and the discharge blood glucose values of SSI were also higher than BBI (SSI 244.3; BBI 128.6; $\mathrm{p}<.001$ ). There were no statistically significant difference on fasting, mean, and discharge blood glucose values. The second goal was to investigate the association of BBI and SSI patients and their LOS. The LOS for SSI was 4.6 days while the BBI was 4.8 days; the results were not statistically significant. The third goal was to determine if there were statistically significant differences between SSI and BBI on episodes of hypoglycemia (mild, moderate, or severe). Hypoglycemia was defined as blood glucose not lower than $70 \mathrm{mg} / \mathrm{dl}$ or $389 \mathrm{mmol} / \mathrm{l}$. The results were not statistically significant. The strength of the study was the results that indicated the SSI group 
had higher fasting, mean and discharge blood glucose values in comparison to the BBI group.

In another classic study, Umpierrez et al. (2013) looked at T2DM patients using $\mathrm{BBI}$ and basal plus bolus in a six-hospital multicenter organization on the East Coast. They enrolled 735 adult patients from the medical-surgical units using the following criteria: (a) patients with blood glucose between 140 and $400 \mathrm{mg} / \mathrm{dl}$ and (b) history of T2DM more than 3 months. The study included two outcome measures to identify the differences in blood glucose levels of patients treated with BBI, basal plus bolus, and SSI. Investigators compared the baseline of clinical characteristics and used the Wilcoxon test to compare continuous variables and chi-squares for distinct variables. Umpierrez et al. determined that there were no significant differences among groups in terms of age, ethnicity, body mass index, or length of stay in the hospital. The results revealed improved blood glucose control and fewer treatment errors in BBI and basal plus bolus as compared to the SSI group. A limitation of the study was that it did not distinguish among the six hospitals. The strength of this study was to show better glycemic control in hospitalized patients receiving BBI and basal plus bolus as compared to SSI. This project informed the ADA's (2016) decision to strongly discourage only the use of sliding scale insulin only in patients with T2DM.

Several studies demonstrated the clinical benefits (Fahim et al., 2015) and better glycemic control of using BBI over SSI (Roberts \& Godbole, 2015; Rymaszewski \& Breakwell, 2013; Umpierrez et al., 2013). For example, the SSI patients who missed their insulin doses or received higher medication doses had more severe hyperglycemic events (Umpierrez et al., 2013). The aim of this study was to contribute to the literature of evidence-based practice on the positive clinical outcomes of hospitalized patient glycemic control. 


\section{CHAPTER 3: METHODOLOGY}

\section{Research Design}

The study was a descriptive quantitative chart review within a community hospital organization. The design was a retrospective closed case review. This study was cross-sectional with data obtained from electronic medical records from May 2017 to May 2018.

\section{Setting}

The study was conducted in a Northern California community-based hospital. The majority of the population is upper middle class and well educated. The hospital serves an ethnically diverse population. The setting was multi-site as there were two medical-surgical units at the same organization. The rationale for choosing two campuses was due to the difference in the average number of patients at each campus. One-unit averaged 16 patients per day and one-unit averaged 80 patients per day. The hospital was familiar with the basal bolus insulin recommendations from the ADA.

\section{Sample}

Inpatient electronic medical records of adult male and female patients with T2DM were analyzed for glycemic control. The sample included 40 electronic records for BBI and 50 electronic records for SSI patients conducted as a retrospective closed medical chart review. The inclusion criteria were as follows: (a) adult patients with known T2DM on admission, (b) T2DM patients who received SSI, (c) T2DM patients who received BBI, and (d) T2DM patients with the length of stay (LOS) of fewer than 5 days discharged from medical-surgical units. The exclusion criteria were as follows: (a) deceased T2DM patients, (b) 
patients with gestational diabetes, (c) pediatric populations, (d) Type 1 diabetes mellitus patients, and (e) T2DM patients who were on observation status less than 24 hours stay. The sources were obtained from review of medical records as part of a retrospective chart review (T2DM patients using ICD 10 codes, filtered by discharged units, BBI and SSI). The SSI and the BBI groups were based on medications given to T2DM patients.

\section{Ethical Considerations}

Patients were de-identified and coded for T2DM who received either BBI or SSI. The data were stored in a protected electronic folder that was password accessible only; the investigator was the only person that could access the data. After 1 year, the medical record numbers (MRN) and the coded numbers in the spreadsheet and all data collection sheets were destroyed.

\section{Procedures}

The Institutional Review Board (IRB) at California State University, Fresno, School of Nursing Research Committee and the El Camino Hospital IRB approved this project. A quantitative chart review was conducted on T2DM patients receiving either BBI or SSI and admitted between May 2017 and May 2018. An information technology specialist reviewed electronic medical records a de-identified the data abstracted by coding. Data were not subject to manipulation.

Inclusion and exclusion criteria were applied. Electronic medical records were filtered for a diagnosis of T2DM base on ICD-10 codes. Next, the subjects who (a) received sliding scale insulin and basal bolus insulin or (b) obtained plasma blood glucose values from point-of-care testing with glucometer from 
medical surgical locations were entered onto an electronic spreadsheet. The SSI and the BBI groups were determined on the medications given to T2DM patients. The SSI group selected should be in SSI only sliding scale in the medical record administration (MAR) but for the purpose of the project should not have received any long-acting insulin. Whereas BBI would have had both. Those who received both BBI (with long-acting insulin) and SSI during the same admission were excluded and did not contribute to either group.

The subjects' age in years, gender and length of stay were noted and entered onto an electronic spreadsheet. Point-of-care blood glucose readings were recorded and examined for glycemic control. Finally, blood glucose readings were averaged for the subject's length of stay.

\section{Data Analysis Plan}

De-identified data were collected and transferred to an electronic spreadsheet and then imported into an electronic statistical package. Data were analyzed using descriptive statistics. Descriptive statistics provide brief observations and summaries about the sample that help identify patterns (Conner $\&$ Johnson, 2017). An independent samples $t$ test was used to determine age differences as well as length of stay between the SSI and BBI groups. A chi squared test of association was used to determine if there were gender differences between the SSI and BBI groups.

Point-of-care testing glucose values were averaged by readings obtained during the subject's length of stay. An estimated hemoglobin A1c was calculated based on the average glucose value. The A1c-Derived Average Glucose (ADAG) Study Group (2008) proposed the relationship between A1C and eAG is described by the formula 28.7 X A1c $-46.7=\mathrm{eAG}$ (Nathan, et al., 2008). 
An independent samples $t$ test was used to analyze the average blood glucose and estimated HbA1c among the SSI and BBI groups. The level of statistical significance for this project was $\mathrm{p} \leq .05$. 


\section{CHAPTER 4: RESULTS}

\section{Statistical Analysis}

A total of 90 electronic medical records were selected for the study based on inclusion and exclusion criteria. Electronic medical records were then divided into two insulin administration groups: BBI and SSI. Demographic variables of age, gender and length of stay were compared between the two groups.

For the entire sample, the average age was 63.66 years $(\mathrm{SD}=15.15$ years) and ranged from 24 years to 94 years. The average length of stay was 6.73 days $(\mathrm{SD}=9.71)$ and ranged from 0 to 55 days (see Table 1$)$.

Table 1

Descriptive Statistics for Age and Length of Stay for the Entire Sample

\begin{tabular}{lccccc}
\hline \multicolumn{1}{c}{ Variable } & $N$ & Minimum & Maximum & $M$ & $S D$ \\
\hline Age & 90 & 24 & 94 & 63.66 & 15.51 \\
Length of Stay & 90 & 0 & 55 & 6.73 & 9.71 \\
\hline
\end{tabular}

The variables of age and length of stay did not differ between the BBI and SSI groups (see Table 2). By group, the mean age of the BBI sample was 62.85 ( minimum age $=24$, maximum age $=94$ ). The mean age of the SSI sample was 64.30 (minimum age $=24$, maximum age $=92$ ). As determined by an independent samples $t$ test, the difference in the average age of 1.45 years between the SSI and BBI group was not statistically significant $(t(88)=-0.43, p=.66)$. By group, the mean length of stay for the BBI sample was 7.98 days with a minimum stay of 0 days and a maximum stay of 55 days. The mean length of stay for the SSI sample was 5.80 days with a minimum stay of 0 days and a maximum stay of 38 days. As 
determined by an independent sample $t$ test, the difference of 2.10 days in the average stay between the SSI and BBI group was not statistically significant $(t(88)$ $=1.01, p=.31)$.

Table 2

Descriptive Statistics for Age and Length of Stay by Group BBI SSI

Variable

$\begin{array}{llll}N & \text { Min } \operatorname{Max} & M & \end{array}$

$\begin{array}{llll}N \quad \operatorname{Min} & \operatorname{Max} & \mathrm{S}\end{array}$

\begin{tabular}{lcccccccccc}
\hline Age & 40 & 24 & 94 & 62.85 & 15.75 & 50 & 24 & 92 & 64.30 & 15.45 \\
Length of Stay & 40 & 0 & 55 & 7.90 & 10.85 & 50 & 0 & 38 & 5.80 & 8.71 \\
& & & & & & & & & & \\
\hline
\end{tabular}

For the entire sample, the subjects' gender was $51.1 \%(n=46)$ males and $48.9 \%$ females $(n=44) ; 44.4 \%(n=40)$ assigned to the BBI group and 55.6\% $(n=$ 50) assigned to the SSI group (see Table 3 ).

Table 3

Frequencies and Percentages of Gender for the Entire Sample

\begin{tabular}{lcc}
\hline Demographic Variables & $n$ & $\%$ \\
\hline Gender & 46 & 51.1 \\
Men & 44 & 48.9 \\
Women & 90 & 100.0 \\
Total & & \\
Group & 40 & 44.4 \\
BBI & 50 & 55.6 \\
SSI & 90 & 100.0 \\
Total & &
\end{tabular}


Table 4 showed the BBI sample was composed of $50 \%$ women $(n=20)$ and $50 \%$ men $(n=20)$. The SSI sample was composed of $48 \%$ women $(n=24)$ and $52 \%$ males $(n=26)$. As determined by a chi square test of association, there were no statistically significant differences in gender between the SSI and BBI group $\left(x^{2}(1)=0.03, p=.85\right.$.

Table 4

Frequencies and Percentages of Gender by Group

\begin{tabular}{lcc}
\hline & $B B I$ & SSI \\
\cline { 2 - 3 } Demographic Variable & $n(\%)$ & $n(\%)$ \\
\hline Gender & & \\
Men & $20(50.0)$ & $26(52.0)$ \\
Women & $20(50.0)$ & $24(48.0)$ \\
Total & $40(100.0)$ & $50(100.0)$ \\
\hline
\end{tabular}

There were no statistically significant differences in age, gender and length of stay between the BBI and the SSI groups.

\section{Average Blood Glucose}

An independent-samples $t$ test was conducted to compare average blood glucose between the BBI and SSI groups. There was a statistically significant difference of 27.20 $\mathrm{mg} / \mathrm{dL}$ in average BG for patient between the SSI $(M=175.09, S D=40.56)$ group and the BBI $(M=201.12, S D=61.82)$ group, $t(86)=2.46, p=.01$. There was a higher average blood glucose level for those in the BBI group as compared to those in the SSI group (see Table 5). 


\section{Estimated Hemoglobin A1c}

An independent-samples $t$ test was conducted to compare estimated hemoglobin A1c blood glucose between the BBI and SSI groups. There was a statistically significant difference of $1.03 \%$ found between estimated HbAlc between the BBI group ( $M=8.76$, $S D=2.10)$ and the SSI group $(M=7.72, S D=1.41) ; t(86)=2.76, p=.007$. These results suggest a higher average estimated $\mathrm{HbA1c}$ for those in the BBI group as compared to the SSI group (see Table 5).

Table 5

Independent Samples $t$ Tests for Averages Blood Glucose and Estimated HbAlc

\begin{tabular}{llccccccc}
\hline Variable & Instruction & $N$ & $M$ & $S D$ & $t$ & $P$ & $\begin{array}{c}\text { Mean } \\
\text { Difference }\end{array}$ & $\begin{array}{c}95 \% \text { CI } \\
\text { (Lower, } \\
\text { Upper) }\end{array}$ \\
\hline $\begin{array}{l}\text { Average } \\
\text { BG }\end{array}$ & BBI & 39 & 202.12 & 61.82 & 2.46 & .01 & 27.02 & $5.24,48.11$ \\
& SSI & 49 & 175.09 & 40.56 & & & & \\
$\begin{array}{l}\text { Estimated } \\
\text { HbA1c }\end{array}$ & BBI & 39 & 8.76 & 2.10 & 2.76 & .007 & 1.03 & $0.29,1.78$ \\
& SSI & 49 & 7.72 & 1.41 & & & & \\
\hline
\end{tabular}

Note. $\mathrm{p}=.007$

Results suggest that SSI provides better glycemic control. Specifically, results suggest that when using SSI, the average blood glucose is lower (see Figure 1) and the estimated $\mathrm{HbAlc}$ is lower (see Figure 2) during the subjects stay in the hospital. 


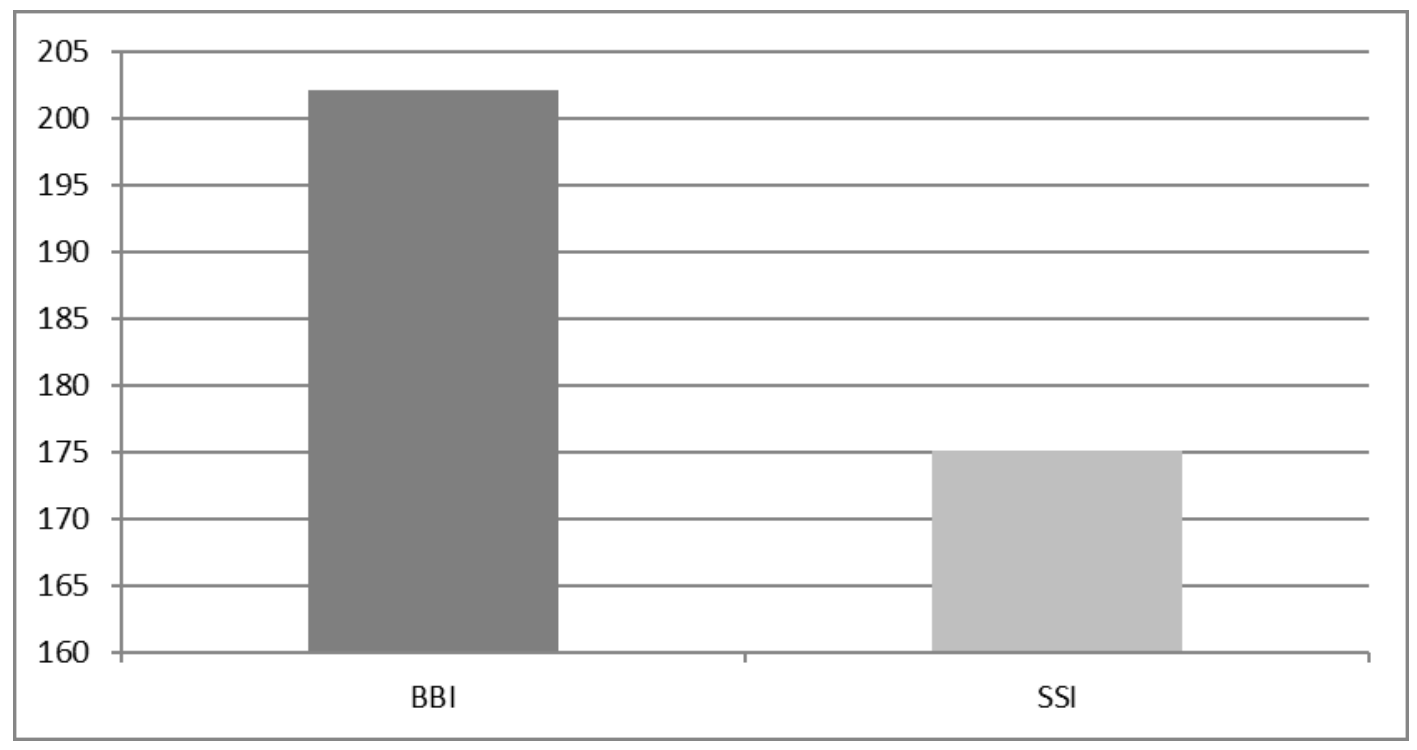

Figure 1. Average blood glucose level mg/dL comparing basal bolus insulin (BBI) to sliding scale insulin (SSI).

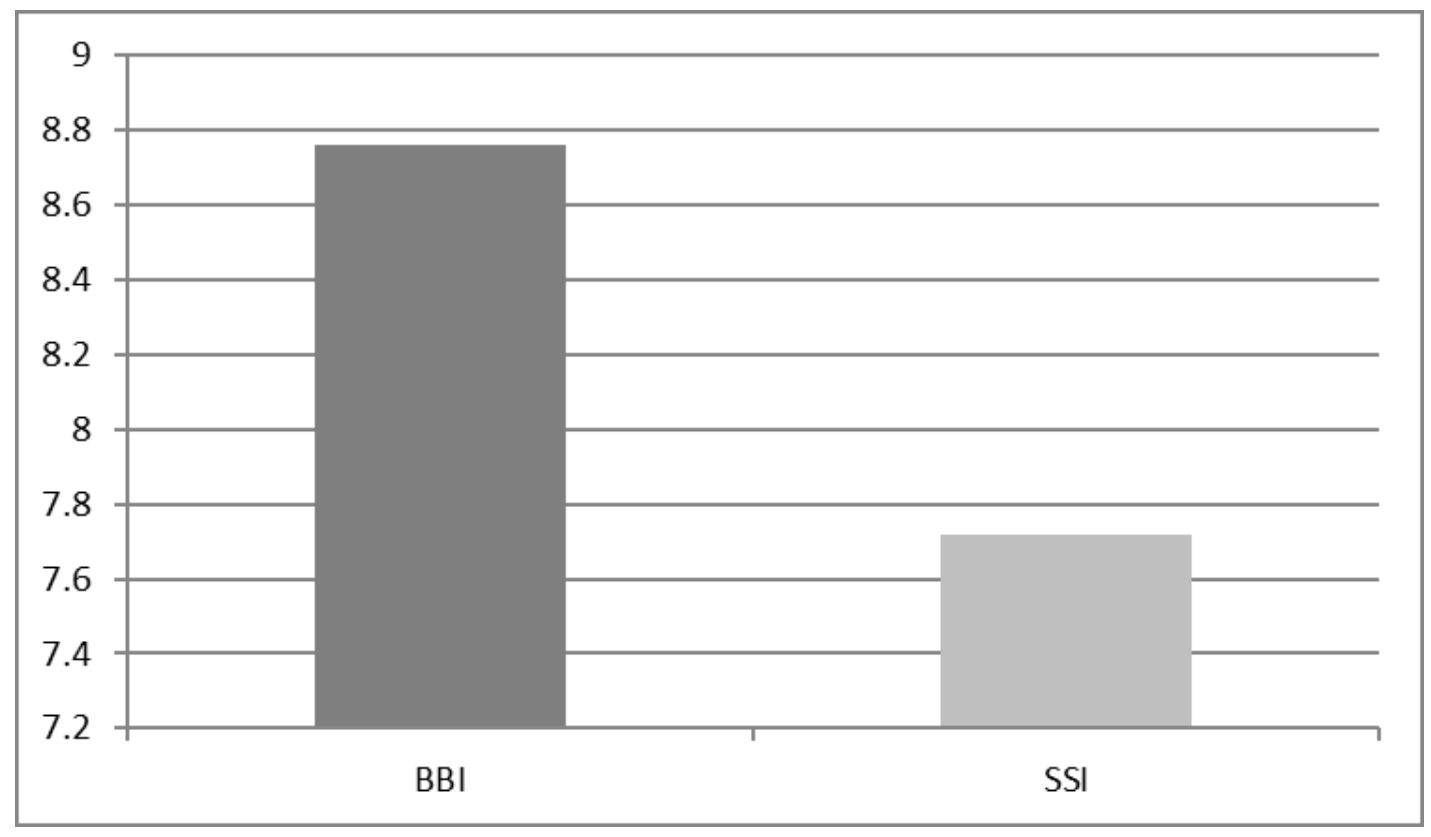

Figure 2. Estimated HbA1c comparing basal bolus insulin (BBI) to sliding scale insulin (SSI) 


\section{CHAPTER 5: DISCUSSION}

This project had many similarities and differences with prior investigations. Between the BBI and SSI groups there were no demographic variable differences between hospitalized patients in this study. In other words, there were no statistical differences between groups as related to age, gender and length of stay which was similar to other literature reviews of Rymaszewski \& Breakwell, 2013, and Umpierrez et al., 2013.

In comparison of the average blood glucose of the two groups, this study revealed that the SSI group showed a lower average blood glucose than the BBI group. This is contrary to several studies which demonstrated the clinical benefits (Fahim et al., 2015) and better glycemic control of using BBI over SSI (Roberts \& Godbole, 2015; Rymaszewski \& Breakwell, 2013; Umpierrez et al., 2013). In fact, Umpierrez et al., 2013 found that SSI patients who missed their insulin doses or received higher medication doses had more statistically significant hyperglycemic events (Umpierrez et al., 2013).

Although the researcher was not able to determine the hypoglycemic events in this study, Rymaszewski and Breakwell (2013) studied the effectiveness of glycemic control with SSI and BBI and found more episodes of mild/moderate hypoglycemic with BBI than SSI therapy, although the difference was not statistically significant. Roberts and Godbole (2015) performed a retrospective chart review of SSI and a prospective chart review of BBI. In the SSI retrospective study, low doses of SSI and BBI were almost the same whereas the SSI group received higher doses of medications than the BBI group.

In comparison with the $\mathrm{HbA1c}$, a statistically significant difference emerged, suggesting a lower average estimated HgbA1c for those in the SSI group 
as compared to the BBI group. In comparison with Rymaaszweski \& Breakwell (2013) study showed HbA1c were not different in BBI and SSI with questionable accuracy in their HbAlc values reported.

Elliott et al. (2016) found that it is also important to use HbA1c as platform targets to understand higher hypoglycemic event rates because patients might present with no symptoms, patients and nurses might not notice no symptoms, and they might underreport events. The study outcomes, which were consistent with Elliott et al.'s (2016) findings, indicated that the retrospective chart review could likely underestimate the real incidence of glycemic control, as compared with the prospective study. The results showed that healthcare professionals did not report lower blood glucose levels as often as higher blood glucose levels (Elliott et al., 2016). Elliot et al.'s (2016) study results revealed the opposite of the stated aim which was to compare the low BG events versus those who are reported in clinical trials. The researchers examined the possibility and why it occurred that way. Elliott et al. (2016) further mentioned that real-world data versus randomized controlled trials (RCTs) can guide healthcare professionals in providing proper management and treatment of choice to achieve glycemic control.

There could be two possible implications in this study for health care providers. First, whether SSI or BBI order sets were used, clinicians need to consider what type of insulin management appropriate for their patients, individualized care based on the patient's glycemic blood levels and presenting signs and symptoms, looking at the patient's overall diagnosis. Another implications, which was not part of this study, to consider clinicians familiarity with SSI and BBI protocols. 


\section{Limitations}

As this study focused on a community hospital organization on two separate units. The results of this descriptive study apply in this setting but are not generalizable. The recent BBI implementation was a small sample size of 90 patients after the initial roll out of a new practice change. Another limitation of the study is its internal validity. The two hospital units have different healthcare providers (e.g., hospitalist, independent clinicians) using either SSI or BBI, and some might find SSI to be more beneficial than BBI. The hospital had undergone new practice changes educating the staff on BBI. Those efforts are ongoing. Finally, the study might not have captured the data documentation of glycemic control completely, especially when limiting or providing more supplemental dextrose or nutritional carbohydrates.

\section{Recommendations for Future Research}

Future research should use prospective study designs for T2DM patients receiving BBI and SSI to understand glycemic control in a hospital setting. In fact, studies that use technology of continuous glucose monitoring would offer more data on glycemic control. This would include more information on hyperglycemic and hypoglycemic events. Future studies could also reveal nurses' levels of comfort and familiarity with BBI. As this study did not include cardiovascular patients with T2DM in the telemetry units, conducting a study of this population is important as T2DM places this population at high risk for co-morbidity associated with poor outcomes if they do not control their glycemic control well (Elliot et.al, 2016). The use of advanced nurse practice nurses and nurse educators/or those who have strong knowledge of and interest in glycemic controls for T2DM inpatients can help improve glycemic control. 


\section{Implications for Nursing Practice}

The results of this study can provide information for nursing. It is important that nurses evaluate their current BBI and SSI protocols and, if necessary, review the guidelines and workflow processes. Nurses should perform evaluations once they have implemented such changes to identify flexibility when modifying guidelines and changing workflow as needed. As this study demonstrated, SSI achieved better glycemic control than BBI; Therefore, more time is needed for evaluating glycemic control and discussing patient outcomes. The use of advanced practice nurses and on-site diabetes nurse educators or resources can provide support not only for nurses, but also for physicians, hospitalists, external providers, by providing inpatient consultation.

\section{Conclusion}

Providing glycemic control for T2DM patients while hospitalized is important. Based on a comparison of average blood glucose levels and the estimated HbA1c the SSI group had better glycemic control than the BBI group. It may be in this community hospital setting that sliding scale insulin was more effective than basal-bolus coverage for glycemic control. More time may be needed to effectively evaluate a new practice change. 
REFERENCES 


\section{REFERENCES}

Alfehaid, L. S., Alotaibi, A. A., Alanazi, A. S., Bustami, R. T., \& El Melik, R. (2018). Clinical outcomes associated with use of subcutaneous sliding scale insulin compared to other insulin regimens in hospitalized patients. International Journal of Clinical Medicine, 9(4), 260-269.

http://dx.doi.org/https://doi.org/10.4236/ijcm.2018.94023

American Diabetes Association (2016). Diabetes care in the hospital. Section 13 in standards of Medical Care in Diabetes-2016. Diabetes Care, 39(Suppl. 1), S99S104.

American Diabetes Association. (2018). Diabetes care in the hospital. Section 14 in standards of medical care in diabetes-2018. Diabetes Care, 41(Suppl. 1), S144S151. doi.org/10.2337/dc18-S014

American Diabetes Association. (n.d.). eAG/A1C conversion calculator. Retrieved from https://professional.diabetes.org/diapro/glucose_calc

Badlani, S., Ford, W. T., \& Yu, D. J., et al. (2014). Evidence for basal-bolus insulin versus slide scale insulin. Current Emergency and Hospital Medicine Reports, 1(2), 26-34. doi.org/10.1007/s40138-013-0032-4

Bertalanffy, L. V. (1972). General system theory: Foundations, development, applications. New York: NY: George Braziller. 
Center for Disease Control [CDC]. (2017). The national diabetes statistics report. Retrieved from https://www.cdc.gov/diabetes/pdfs/data/statistics/nationaldiabetes-statistics-report.pdf

Chandler, J., Rycroft-Malone, J., Hawkes, C., \& Noyes, J. (2015). Application of simplified complexity theory concepts for healthcare social systems to explain the implementation of evidence into practice. Journal of Advanced Nursing, 72(2), 461-480. http://dx.doi.org/10.1111/Jan.12815

Conner, B., \& Johnson, E. (2017, November). Descriptive statistics: Use these tools to analyze data vital to practice-improvement projects. American Nurse Today, 12, $52-55$.

Cooper, H. C., Geyer, R. (2009). What can complexity theory do for diabetes management? Linking theory to practice. Journal of Evaluation in Clinical Practice, 15(4), 761-765. http://dx.doi.org/10.1111/j.1365-2753.2009.01229.x

Elliott, L., Fidler, C., Ditchfield, A., \& Stissing, T. (2016). Hypoglycemia event rates: A comparison between real-world data and randomized controlled trial populations in insulin-treated diabetes. Diabetes Therapy, 7(1), 45-60. http://dx.doi.org/10.1007/s13300-016-0157-z

Fahim, G., Davanos, E., Muzykovsky, K., \& Rublin, R. (2015). Pilot and revision of a basal-bolus dosing guideline for the management of hyperglycemia in noncritically ill adult patients. Journal of Pharmacy Practice, 28(6), 504-510. http://dx.doi.org/10.1177/0897190014544818 
Harris, S., Narayan, K., \& Ali, M. K. (2015). Quality improvement in diabetes: Successful in achieving better care with hopes of prevention. Annals of The New York Academy of Sciences, 138-151. http://dx.doi.org/10.1111/nyas.12950

Klonoff, D. C. (2014). Point-of-care blood glucose meter accuracy in the hospital setting. Diabetes Spectrum : A Publication of the American Diabetes Association, 27(3), 174-179. http://dx.doi.org/10.2337/diaspect.27.3.174

Johnson, J. A., \& Van Horn, E. R. (2011). The effects of correction insulin and basal insulin on inpatient glycemic control. MEDSURG Nursing, 20(4), 187-193.

Litaker, D., Tomolo, A., Liberatore, V., Stange, K., \& Aron, D. (2006). Using complexity theory to build interventions that improve health care delivery in primary care. Journal General Internal Medicine, 21(S2), S30-S34. http://dx.doi.org/10.1111/j/1525-1497.2006.00360.x

Melnyk, B. M., Morrison-Beedy, D., \& Cole, R. (2015). Generating evidence through quantitative research. In B. M. Melynk \& E. Fineout-Overholt (Eds.), Evidencebased practice in nursing \& healthcare: A guide to best practice (3 ed., pp. 439475). Philadelphia PA: Wolters Kluwer.

Nathan, D., Kuenen, J., Borg, R., Zheng, H., Schoenfeld, D., \& Heine, R. (2008). Translating the $\mathrm{A} 1 \mathrm{C}$ assay into estimated average glucose values. Diabetes Care, 31(8), 1473-8.

National Quality Forum [NQF]. (n.d.). http://www.qualityforum.org/

Roberts, G. W., \& Godbole, G. P. (2015). The impact of basal bolus and sliding scale insulin management on dosing administration errors in hospital setting. Journal of 
Pharmacy Practice and Research, 45(2), 272-275.

http://dx.doi.org/10.1002/jppx.1134

Rymaszewski, H. L., \& Breakwell, S. (2013, April). A retrospective review of sliding scale vs. basal/bolus insulin protocols. The Journal for Nurse Practitioners, 9(4), 214-218. http://dx.doi.org/10.1016/j.nurpra.2013.03.001

Skyttner, L. (2006). General systems theory: problems, perspectives, practice (2 ed.). Retrieved from https://ebookcentral.proquest.com

Tuffin, R. (2016). Implications of complexity theory for clinical practice and healthcare organization. British Journal of Anesthesias Education, 16(10), 349-352. http://dx.doi.org/10.1093/bjaed/mkw013

Umpierrez, G. E., Smiley, D., Hermeyer, K., Khan, A., Olson, D. E., Newton, C., ... Fonseca, V. A. (2013, August). Randomized study comparing a basal-bolus with a basal plus correction insulin regimen for the hospital management of medical and surgical patients with type 2 diabetes: Basal bolus trial. Diabetes Care, 36(8), 2169 -2174. http://dx.doi.org/10.2337/dc12-1988

Umpierrez, G. E., Smiley, D., Jacobs, S., Temponi, A., Mulligan, P., Umpierrez, D., ... Rizzo, M. (2011). Randomized study of basal-bolus insulin therapy in the inpatient management of patients with type 2 diabetes undergoing general surgery (rabbit 2 surgery). Diabetes Care, 34(2), 256-261. http://dx.doi.org/10.2337/dc101407

Umpierrez, G. E., Smiley, D., Zisman, A., Prieto, L., Palacio, A., Ceron, M., ... Mejia, R. (2007). Randomized study of basal-bolus insulin therapy in the inpatient 
management of patients with type 2 diabetes (rabbit 2 trial). Diabetes Care, 30(9), 2181 -2186. http://dx.doi.org/10.2337/dc07-0295 
APPENDICES 
APPENDIX A: EL CAMINO HOSPITAL IRB APPROVAL 


\author{
Institutional Review Board \\ 2500 Grant Road (ECH-1C35) \\ Mountain View, CA 94040
}

July 9,2018

Meriam Signo, RN

Principal Investigator

RE: $\quad$ ECH 18-10: The Effect of Implementation of Basal-Bolus Insulin Administration on Hypoglycemic Events in patients with Type 2 Diabetes Mellitus at a Community Hospital

Items Reviewed: Request for Approval Application and Questionnaire, Protocol, Waiver Request Form, Data Collection Tools, Literature Review, Research Team Conflict of Interest Statements, NIH Training Certificates, CVs

Dear Ms. Signo:

The El Camino Hospital Institutional Review Board ( $I R B)$ would like to thank you for submitting the documents noted above for the above-captioned research. On behalf the IRB I have reviewed and noted the following:

The purpose of this study is to retrospectively track the frequency of hypoglycemic blood sugar readings between T2DM patients managed with BBIA versus SSI. The research question posed is whether for Type 2 Diabetes Mellitus medical-surgical inpatients, are there fewer hypoglycemic episodes for patients who received basal-bolus insulin compared to sliding scale insulin during their hospital stay? All data collected for this study will be retrospective, no subjects will be enrolled prospectively.

The submission was revised by the PI as follows:

1) Ratio of total number of low readings over the total number of readings has been added as part of the data collection process/procedure.

2) Selecting frequency as number of hypoglycemic episodes, added to protocol.

3) Readings while on basal bolus or while on sliding scale or switch to either one has been added to collection data procedure and collection tool kit,

4) Hypoglycemia blood glucose levels categorized and added to protocol and collection tool

5) Added average $B G$ readings during patient stay while on basal bolus versus sliding scale. This will be part of data collection process

6) Removal of duplicate readings of results. Duplication is not value added time.

7) Hemoglobin $\mathrm{AlC}$ values have been added to the collection process and tool. Good point!

8) For patients longer or shorter period of stay in the hospital, PI added this to the inclusion criteria and part of collection data. 
Ms. Signo

Page 2

The Privacy Rule (HIPAA law) requires that certain criteria be met before a waiver of consent and authorization to collect data for research can be granted by the IRB. The criteria for waiver is met as follows:

- No identifying subject information will be collected in the research dataset.

- Data collection will be from May 2017 and May 2018 for routine standard care services. All patients have been discharged and are no longer being followed.

- Upon completion of the study, the MRN and coded numbers in the spreadsheet and all data collection sheets will be destroyed. The DNP program requires proposal project to be submitted for publication and there is a chance for this study to be published. Only aggregate data and results will be incorporated in any publications.

Based on review of the materials as outlined above, on behalf of the $\mathbb{R B}$, I have determined that this research meets the criteria to qualify for waiver of patient authorization and consent. In addition, this research qualifies for expedited review, therefore approval is granted on July 9,2018. You can now feel free to proceed with your research study, subject to review in one (1) year. The full board will be notified of this action at the next meeting.

The Board's continued approval is contingent on the following:

1. Your compliance with the approved investigational plan.

2. Your use of the approved consent form.

3. Your commitment to notify the Board of any unanticipated events or complications that might reasonably have an effect on the Board's continued approval of the study including breach of confidentiality.

Study Expiration Date: July 8, 2019

Sincerely,

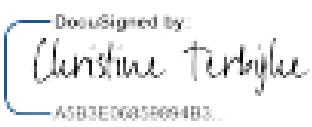

Christine Terbijhe, on behalf of Michael Greenfield, MD

Chairman - Institutional Review Board 
APPENDIX B: CALIFORNIA STATE UNIVERSITY, FRESNO IRB APPROVAL 


\title{
FRESN@STATE \\ College of Health and Human Services
}

\author{
California State University, Fresno \\ School of Nursing \\ IRB Approval
}

Date: August 3, 2018

RE: DNP 1805 The Effect of Implementation of Basal-Bolus Insulin on Hypoglycemic Events on Inpatients at a Community Hospital

Dear Meriam Signo,

As the Chair of the School of Nursing Research Committee, serving as the Institutional Review Board for the School of Nursing, I have reviewed and approved your review request for the above-referenced project for a period of 12 months. I have determined your study to meet the criteria for Minimal Risk IRB review.

Under the Policy and Procedures for Research with Human Subjects at Califormia State University, Fresno, your proposal meers minimal risk criteria according to section 3.3.7: Research in which the risks of harm anticipated are not greater, probability and magnitude, than those ordinarily encountered in daily life or during the performance of routine physical or psychological examinations or tests.

The Research Committee may periodically wish to assess the adequacy of research process.

If, in the course of the study, you consider making any changes in the protocol or consent form, you must forward this information to the Research Committee prior to implementation unless the change is necessary to eliminate an apparent immediate hazard to the research participant(s).

This study expires: August 3, 2019

The Research Committee is authorized to periodically assess the adequacy of the consent and research process. All problems having to do with subject safety must be reported to the Research Committec. Please maintain proper data control and confidentiality. - $*$

If you have any questions, please contact me through the CSU, Fresno School of Nursing Research Committee at symiller@csufresno.edu.

Sincerely,

Suluaidilu Sylvfa Miller, EdD, RN, FNP-C

School of Nursing, Research Committee, Chair

School of Nursing

California State Uniwersity, Fresno - Mclane Hall, floom 190

2345 East San Ramon Ávenue M/S MH25 - Fresna, California 93740-8031

P 559.278.2041 F 559.278.6360 www.FresnoState.edu/chhs/nursing 